\title{
The Dynamic Innovative Balance System Improves Balance Ability: A Single Blind, Randomized Controlled Study
}

\author{
Bunyamin Haksever, PT, PhD $^{1}$ (D) ${ }^{\text {, }}$, Irem Duzgun, PT, PhD² (D), Gul Baltaci, PT, PhD ${ }^{3}$ (D) \\ 1 Physiotherapy and Rehabilitation, Faculty of Health Sciences, Cyprus International University, ${ }^{2}$ Sports Physiotherapy Unit, Faculty of Physiotherapy \\ and Rehabilitation, Hacettepe University, ${ }^{3}$ Physical Therapy and Rehabilitation, Private Ankara Guven Hospital \\ Keywords: exercise, balance, performance, function, assessment
}

https://doi.org/10.26603/001c.25756

\section{International Journal of Sports Physical Therapy}

Vol. 16, Issue 4, 2021

\section{Background}

Functional balance training is crucial for both rehabilitation and prevention. A Dynamic Innovative Balance System (DIBA) is readily available for utilization in both functional and postural control training in a wide variety of dynamic conditions.

\section{Purpose}

The purpose of this study was to compare the effectiveness of the DIBA and standard balance training tools on dynamic and static balance.

\section{Study Design}

Randomized controlled trial

\section{Methods}

Thirty-six healthy males (18 to 32 years) were randomly assigned to group DIBA ( $\mathrm{n}=18)$ or to the control group $(n=18)$ who performed balance training using a balance board, a wobble board, the BOSU, or a soft cushion block for eight weeks. Each participant was assessed before training, at the end of the fourth and eighth week by using the Flamingo balance test (FBT) for assessing static balance ability and using Y-Balance Test (YBT) for dynamic balance ability.

\section{Results}

No significant differences were found in FBT and YBT between the DIBA and control groups at the end of fourth week $(\mathrm{p}>0.05)$. However, at the end of the eighth week, the DIBA group demonstrated statistically significantly better balance ability on the anterior component of YBT $(p=0.001)$ and FBT $(p=0.024)$ than controls.

\section{Conclusion}

The results of this study suggest that the DIBA was effective in both static and dynamic balance training and it may be used alongside other balance tools in a clinical setting. Further studies should include in lower extremity problems to confirm that DIBA training adaptations are transferred to clinical improvements in performance and balance qualities.

\section{Level of Evidence}

$2 d$

\footnotetext{
a Corresponding author: Bunyamin Haksever PT, PhD

Cyprus International University, Faculty of Health Sciences, Dept of Physiotherapy and Rehabilitation, Haspolat, Nicosia, Turkey E-mail:yasbun@hotmail.com Tel: 90-536-2666969
} 


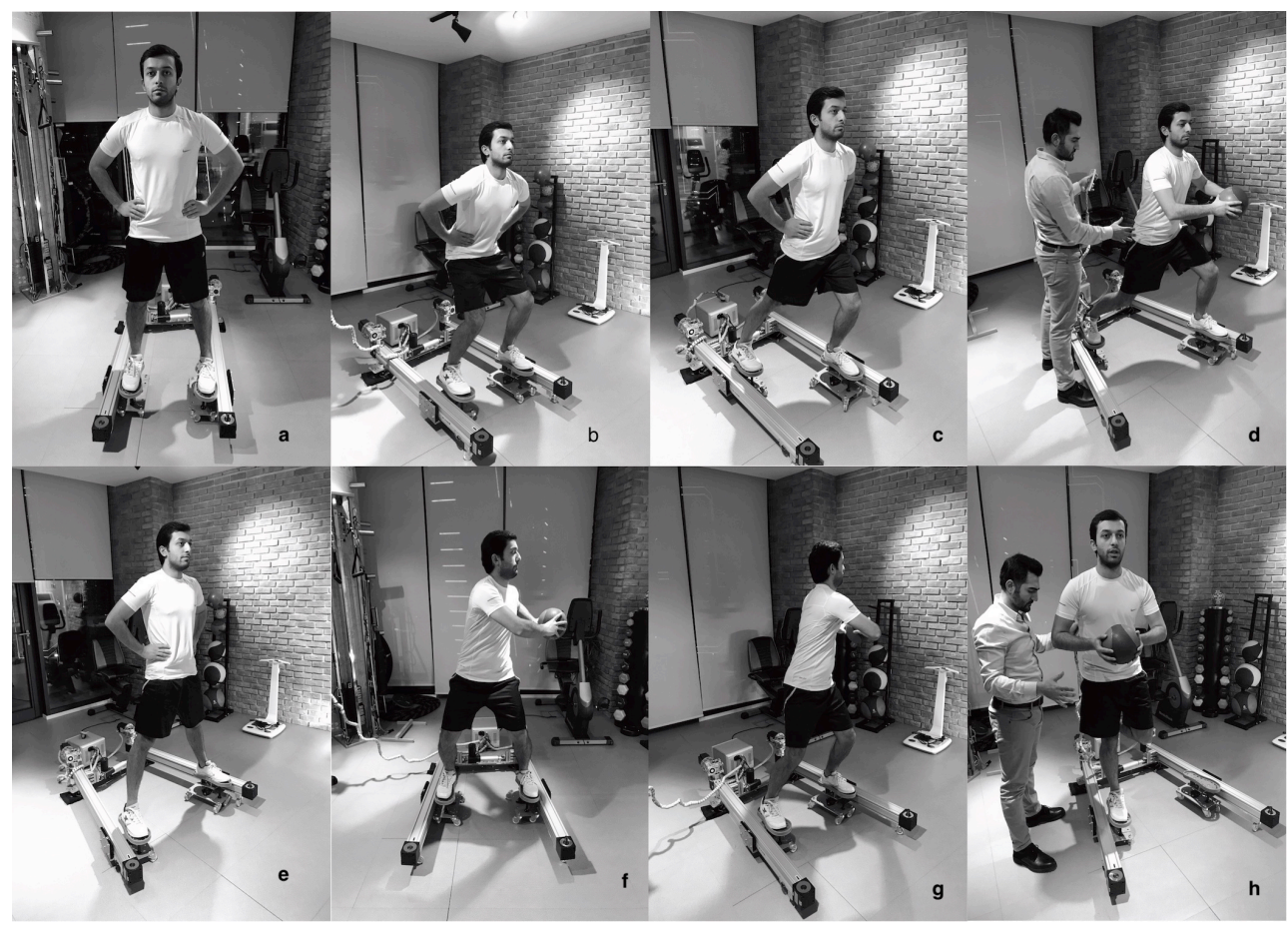

Figure 1. Examples of exercises being performed on the Dynamic Innovative Balance (DIBA) system.

a. steady stance, b. squat, c. lunge, d. squatting in lunge position, e. steady stance while foot platforms move away and together, f. upper body rotation, g. upper body rotation while foot platforms move away and together, g. single leg stance while catching a ball.

\section{INTRODUCTION}

In the last decade, several balance systems have been used for functional training in balance and postural control during rehabilitation and return to sports. Accurate control of posture and balance depends on a correct motor command, which in turn relies on vestibular, visual, and proprioceptive inputs. ${ }^{1-4}$

Balance and functional exercise interventions are essential parts of a rehabilitation program to improve balance and kinesthetic sense of body parts in order to prevent injury recurrence. ${ }^{1,2}$ Most of current balance training systems provide training over a static foot, placed on different surfaces..$^{5}$ According to published literature, the BOSU, ${ }^{6}$ wobble board, ${ }^{7,8}$ rocker board, and virtual reality systems like Nintendo Wii ${ }^{8-10}$ are used for functional balance training. However, patients may have avoided putting the necessary body weight on the injured leg during bilateral stance while using such systems during the training program. This reduces the motivation of a patient because they may fear re-injury or aggravation of their pain. ${ }^{11,12}$ Despite many balance training systems having been described in the literature, few provide dynamic training options and promote sufficient weight shift to the injured side.

The Dynamic Innovative Balance (DIBA) system consists of two mobile foot platforms that independently move in antero-posterior and medio-lateral directions. This mechanism is provided with a remote control, which gives advantages for control of the device for change(s) of direction. This system allows the imitation of movements that occur during functional daily activities. The subject being trained places his/her feet on the platforms and attempts to main- tain his / her balance in different positions such as standing, walking, squatting, and lunging on the moving foot platforms in pre-determined directions. (Figure 1) Balance training exercises are selected among a series of exercise and training protocols, which have been developed for use with the DIBA.

To the authors' knowledge, there is no published literature examining any balance exercise or training protocols with the DIBA in clinical settings. Therefore, the purpose of this study was to compare the effectiveness of the DIBA and standard balance training tools on dynamic and static balance. It was hypothesized that balance exercises and training with DIBA as effective as exercising with standard balance tools.

\section{METHODS}

\section{PARTICIPANTS}

A total of 36 healthy males with age ranging between 18-32 years participated in this study. Participants were randomly assigned into two groups as DIBA group $(\mathrm{n}=18)$ and control group $(n=18)$. For allocation of the participants, a computer-generated list of random numbers was used.

The University Institutional Review Board approved the ethical protocol for this study, and all volunteers were informed about the nature of the study and signed a written consent form. Flow chart of the study is shown in Figure 2.

Inclusion criteria included: males, age $18-40 \mathrm{yrs}$, with no lower extremity injury in the prior six months, no chronic pain or surgery in lower extremity, right lower extremity dominant, and willing to participate to the study as a volunteer. Participants who had any neuromuscular, cardiores- 
piratory, neurologic disorders, had sustained any musculoskeletal injuries over the prior six months, were currently experiencing pain anywhere in the body, and had not participated in three training sessions or two assessment sessions, or had pain that could interfere with the training and assessment sessions were excluded from this study. The participants were also advised not to consume alcohol, take nutritional supplements, participate in physical activities, or use other recovery techniques such as analgesic drugs and cryotherapy, throughout this study. Moreover, they were asked to maintain their usual nutritional and water intake over the course of this study. Participants, who met inclusion criteria were randomly chosen among initially assessed 48 healthy males, who performed moderate intensity exercise lasting from 30 to 60 minutes at least three days a week based on the criteria of American College of Sport Medicine. ${ }^{13,14}$

\section{PROCEDURE}

Balance assessment: Both the DIBA and control groups were assessed before balance training, and again at the end of the $4^{\text {th }}$ and $8^{\text {th }}$ weeks. The Y-Balance test (YBT) was employed for dynamic balance assessment and Flamingo balance test (FBT) was used for static balance assessment as described elsewhere. ${ }^{15,16}$ Each test was repeated three times consecutively using the dominant lower extremity (all participants were right-side dominant), and the average value of three measurements was used for statistical analysis.

The DIBA uses an electromechanical dynamic balance training system, which developed by the authors. It consists of two-foot platforms that move on its rail. The rails also are able to move mediolaterally to increase or decrease distance between the feet for changing exercise and training load.

Balance training program: All participants performed balance training supervised by same physical therapist at the same clinic. Each training session was set 45-60 minutes, three days per week for eight weeks in total. Although, both groups received different types of exercise, the intensity of exercises was similar. Exercises for the DIBA group were divided into three categories according to their intensity. The low intensity exercises were completed during the first two weeks, moderate intensity exercises in the $3^{\text {rd }}$ to $4^{\text {th }}$ weeks, and high intensity exercises were completed during the $5^{\text {th }}$ to- $8^{\text {th }}$ weeks. Balance sample exercises for the DIBA are shown in Figure 1.

Duration, repetitions and number of sets are given Table 1

Exercise protocol for control group: Participants in the control group were received common balance exercises using BOSU, balance boards, wobble board, and a soft cushion block. Exercise duration was 45-60 minutes, three days per week for eight weeks in total.

\section{STATISTICAL METHODS}

Data were analyzed using IBM SPSS v.22 (IBM, Chicago, IL, USA). Schapiro Wilk test was employed for whether data were normally distributed. As data were not normally distributed, Mann Whitney U test was used for between group comparisons, and Wilcoxon Signed Rank test with Bonfer-

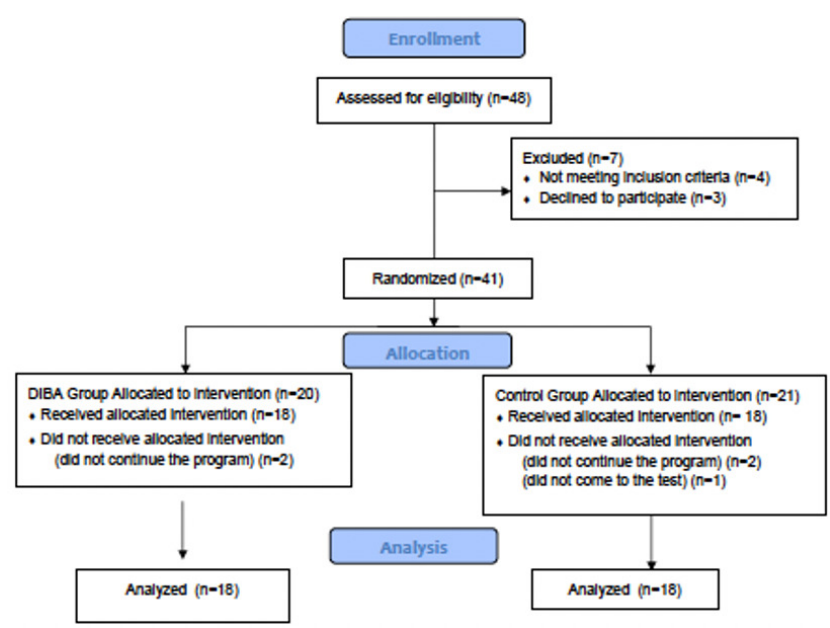

Figure 2. Flow diagram of randomized controlled trial.

DIBA= Dynamic Innovative Balance System.

roni correction was employed for within group comparisons. The number of subjects in the study was based on the anterior parameter of the YBT test, for the power of $80 \%$, and an alpha value of $5 \%$, which indicated that at least 18 subjects should be included for each group.

\section{RESULTS}

The demographic data is shown in Table 2. Both the groups were not statistically significantly different in age, body weight, body height, and body mass index $(p>0.05)$.

There were no significant differences between groups in scores on the components of the YBT $(p>0.05)$ at baseline and at the end of $4^{\text {th }}$ week measurements. However, a statistically significant difference was seen for only anterior component of YBT $(p=0.001)$ in favor of the DIBA group at the end of the $8^{\text {th }}$ week. Within group comparisons demonstrated significantly higher YBT scores in the anterior $(p=0.01)$ and posteromedial $(p=0.014)$ directions at the end of $8^{\text {th }}$ week compared with baseline scores for the DIBA group (Figure 3 ). Similarly, the scores of postero-medial $(\mathrm{p}=0.008)$ and postero-lateral (0.001) components of the YBT were significantly higher at the end of $8^{\text {th }}$ week compared with those of baseline measurement for control group (Table 3).

There was not a statistically significant difference between groups in scores of the FBT at baseline and at the end of $4^{\text {th }}$ week ( $\left.p>0.05\right)$; however, the number of falls was significantly lower in favor of the DIBA group $(\mathrm{p}=0.024)$ at the end of the $8^{\text {th }}$ week. The DIBA group demonstrated a statistically significant lower number of falls during FBT at the end of $8^{\text {th }}$ week $(p=0.011)$ compared to those at baseline measurement. There was not a statistically significant difference within group FBT scores ( $p>0.0167$; Bonferroni correction) in control group (Figure 4 ).

\section{DISCUSSION}

The purpose of this study was to investigate whether results 
Table 1. Duration, repetitions and number of sets in balance exercises for DIBA and Control Group*

\begin{tabular}{|c|c|c|c|}
\hline Weeks & Balance Training Program & $\begin{array}{l}\text { DIBA group } \\
\text { Time } X \text { repetition } X \text { set }\end{array}$ & $\begin{array}{l}\text { Control group } \\
\text { Time } X \text { repetition } X \text { set }\end{array}$ \\
\hline \multirow[t]{6}{*}{$0-2$ weeks } & 1: Steady standing in upright position * & $60 \mathrm{~s} \times 8$ rep. & $60 \mathrm{~s} \times 8$ rep. \\
\hline & $\begin{array}{l}\text { 2: Steady standing in upright position on DIBA with } \\
\text { balance }\end{array}$ & 60 s $\times 8$ rep. & 60 s $X 8$ rep. \\
\hline & 3: Steady standing in the squat position * & 30 s $\times 10$ rep. & 30s X 10 rep. \\
\hline & 4: Steady standing in the squat position on DIBA & 30s $\times 10$ rep. & 30s X 10 rep. \\
\hline & 5: Squatting exercise in lunge position ${ }^{*}$ & 10 rep. $X 3$ set & 10 rep. $X 3$ set \\
\hline & 6: Squatting exercise in lunge position on DIBA & 10 rep. $X 3$ set & 10 rep. $X 3$ set \\
\hline \multirow[t]{6}{*}{$3-4$ weeks } & $\begin{array}{l}\text { 7: Catching and throwing ball in steady lunge position on } \\
\text { DIBA and on floor* }\end{array}$ & 15 rep. $X 3$ set & 15 rep. $X 3$ set \\
\hline & $\begin{array}{l}\text { 8: Catching and throwing ball in steady squat position on } \\
\text { DIBA and on floor* }\end{array}$ & 30s $\times 10$ rep. & 30s X 10 rep. \\
\hline & $\begin{array}{l}\text { 9: Steady standing in upright position while DIBA foot } \\
\text { platforms move away and toward each other. }\end{array}$ & 12 rep. $X 3$ set & 12 rep. $X 3$ set \\
\hline & $\begin{array}{l}\text { 10: Squatting exercise as DIBA foot platforms move away } \\
\text { from and toward each other. }\end{array}$ & 12 rep. $X 3$ set & 12 rep. $X 3$ set \\
\hline & $\begin{array}{l}\text { 11: Steady standing in squat position with eyes closed on } \\
\text { DIBA and on floor*. }\end{array}$ & 30s X 10 rep. & 30s X 10 rep. \\
\hline & $\begin{array}{l}\text { 12: Steady standing in lunge position with eyes closed. on } \\
\text { DIBA and on floor* }\end{array}$ & 30s X10 rep. & 30s X 10 rep. \\
\hline \multirow[t]{6}{*}{$5-8$ weeks } & $\begin{array}{l}\text { 13: Rotation of upper body as foot platforms move away } \\
\text { from and toward each other on DIBA and on floor*. }\end{array}$ & $\begin{array}{l}\text { All direction } 8 \\
\text { repX3set }\end{array}$ & $\begin{array}{l}\text { All direction } 8 \\
\text { repX3set }\end{array}$ \\
\hline & $\begin{array}{l}\text { 14: Lunge exercise as foot platforms move antero- } \\
\text { posteriorly while they away from each other and on floor } \\
* \text {. }\end{array}$ & 15 rep.X 3 set & 15 rep.X 3 set \\
\hline & $\begin{array}{l}\text { 15: Single leg stances foot platforms move away from } \\
\text { and toward each other and antero-posteriorly on floor*. }\end{array}$ & 30s X 12 rep. & 30 s $X 12$ rep. \\
\hline & $\begin{array}{l}\text { 16: Catching a ball during single leg stance on foot } \\
\text { platforms as move away from and toward each other and } \\
\text { antero-posteriorly on floor*. }\end{array}$ & 30s $X 12$ rep. & 30s X 12 rep. \\
\hline & $\begin{array}{l}\text { 17: Single leg stance with eyes open as foot platforms } \\
\text { move away from and toward each other and antero- } \\
\text { posteriorly on floor*. }\end{array}$ & 20 s $X 8$ rep. & 20 s $X 8$ rep. \\
\hline & $\begin{array}{l}\text { 18: Single leg stance with eyes closed as foot platforms } \\
\text { move away from and toward each other and antero- } \\
\text { posteriorly on floor*. }\end{array}$ & 20 s $X 8$ rep. & 20 s $X 8$ rep. \\
\hline
\end{tabular}

s: second; Rep: repetition; * = exercises performed by the control group

Table 2. Demographic characteristics of the subjects

\begin{tabular}{|c|c|c|c|}
\hline & $\begin{array}{l}\text { DIBA group }(n=18) \\
\text { Mean } \pm S D \text { (Range) }\end{array}$ & $\begin{array}{l}\text { Control group }(n=18) \\
\text { Mean } \pm S D \text { (Range) }\end{array}$ & $\mathrm{p}$-value \\
\hline Age (year) & $24.4 \pm 3.3(18-32)$ & $23.7 \pm 2.8(20-29)$ & 0.525 \\
\hline Body Height $(\mathrm{cm})$ & $178.2 \pm 5.0(168-188)$ & $176.1 \pm 5.7(169-176)$ & 0.253 \\
\hline Body Weight (kg) & $73.2 \pm 6.9(62-85)$ & $70.8 \pm 6.7(58-82)$ & 0.314 \\
\hline Body Mass Index $\left(\mathrm{kg} / \mathrm{m}^{2}\right)$ & $22.7 \pm 1.9(19-25)$ & $22.4 \pm 2.6(17-26)$ & 0.720 \\
\hline
\end{tabular}

of balance exercises using the DIBA differ from those of balance training using standard balance exercises tools such as BOSU, balance boards, wobble board, and a soft cushion block. Following the eight-week balance training and exercise intervention, the DIBA group reached a greater distance on the anterior component of YBT, and had a lower 
Table 3. Between and within group comparison of Y-Balance Test scores.

\begin{tabular}{|c|c|c|c|}
\hline Direction & DIBA group Mean \pm SD (Range) & Control group Mean \pm SD (Range) & $p$-value \\
\hline \multicolumn{4}{|l|}{ Anterior (cm) } \\
\hline Baseline & $77.0 \pm 5.8(66-87)$ & $74.1 \pm 5.5(64-86)$ & 0.134 \\
\hline $4^{\text {th }}$ week & $77.3 \pm 6.2(67-90)$ & $75.2 \pm 5.4(66-86)$ & 0.293 \\
\hline $8^{\text {th }}$ week & $81.0 \pm 6.0(66-90)$ & $74.7 \pm 4.8(68-86)$ & $0.001^{* *}$ \\
\hline$p$-values & $\begin{array}{l}>0.0167 \text { (Baseline vs. 4th wk) } \\
=0.01 \text { (Baseline vs. 8th wk)** } \\
>0.0167 \text { (4th. week vs. 8th. week) }\end{array}$ & $\begin{array}{l}>0.0167 \text { (Baseline vs. 4th wk) } \\
>0.0167 \text { (Baseline vs. 8th wk) } \\
>0.0167 \text { (4th. week vs. 8th. week) }\end{array}$ & \\
\hline \multicolumn{4}{|c|}{ Posteriomedial $(\mathrm{cm})$} \\
\hline Baseline & $88.6 \pm 9.5(65-109)$ & $85.9 \pm 4.4(78-93)$ & 0.171 \\
\hline $4^{\text {th }}$ week & $88.2 \pm 7.6(68-100)$ & $87.1 \pm 5.0(77-97)$ & 0.406 \\
\hline $8^{\text {th }}$ week & $90.8 \pm 9.8(70-115)$ & $87.8 \pm 5.3(80-95)$ & 0.293 \\
\hline$p$-values & $\begin{array}{l}>0.0167 \text { (Baseline vs. } 4^{\text {th }} \text { wk) } \\
=0.014 \text { (Baseline vs. 8th wk)** } \\
>0.0167 \text { (4th. week vs. 8th. week) }\end{array}$ & $\begin{array}{l}>0.0167 \text { (Baseline vs. 4th wk) } \\
=0.008 \text { (Baseline vs. 8th wk)** } \\
>0.0167 \text { (4th. week vs. 8th. week) }\end{array}$ & \\
\hline \multicolumn{4}{|c|}{ Posteriolateral (cm) } \\
\hline Baseline & $91.1 \pm 8.3(74-108)$ & $88.1 \pm 4.4(88-92)$ & 0.226 \\
\hline $4^{\text {th }}$ week & $90.1 \pm 8.1(74-107)$ & $90.0 \pm 4.5(81-96)$ & 0.988 \\
\hline $8^{\text {th }}$ week & $91.5 \pm 7.7(76-110)$ & $91.6 \pm 4.9(80-98)$ & 0.563 \\
\hline$p$-values & $\begin{array}{l}>0.0167 \text { (Baseline vs. 4th wk) } \\
>0.0167 \text { (Baseline vs. 8th wk) } \\
>0.0167 \text { (4th. week vs. 8th. week) }\end{array}$ & $\begin{array}{l}>0.0167 \text { (Baseline vs. 4th wk) } \\
=0.001 \text { (Baseline vs. 8th wk)** } \\
>0.0167 \text { (4th. week vs. 8th. week) }\end{array}$ & \\
\hline
\end{tabular}

* Significant difference at $\mathrm{p}<0.05$

**Significant difference $\mathrm{p}<0.0167$ (Bonferroni correction)

number of falls on the FBT.

Several authors have suggested that a decrease in lower limb proprioceptive sense after injures is linked with balance deficits. ${ }^{17-19}$ However, both static and dynamic balance may improve with properly designed exercise and training programs. Muscle spindles and Golgi tendon organs are considered the main proprioceptors, ${ }^{20,21}$ and their function may improve with conditioning and strengthening exercises. ${ }^{21-23}$ There are a number of balance training tools and devices currently used in clinical settings for improving muscle strength and proprioceptive sense in order to increase balance ability following lower extremity injury. Few of them have features of computerized or electromechanically controlled mechanisms and the ability to provide customized training programs.

Most systems lack functionality and the ability to simulate activities of daily life. Ground surface balance training tools are generally fixed to the floor. While most balance training equipment does not provide perturbation, which dynamically challenges participants, the DIBA has dual movable foot platforms allowing for perturbation in mediolateral and antero-posterior directions during different postural conditions such as lunging, squatting, single leg stance with eyes open and closed, and also can be used with additional activities such as throwing and catching ball.

Because physical exercise and training for increasing balance ability requires an individual's active participation, ${ }^{17,24}$ the DIBA, with its variety of activity combinations

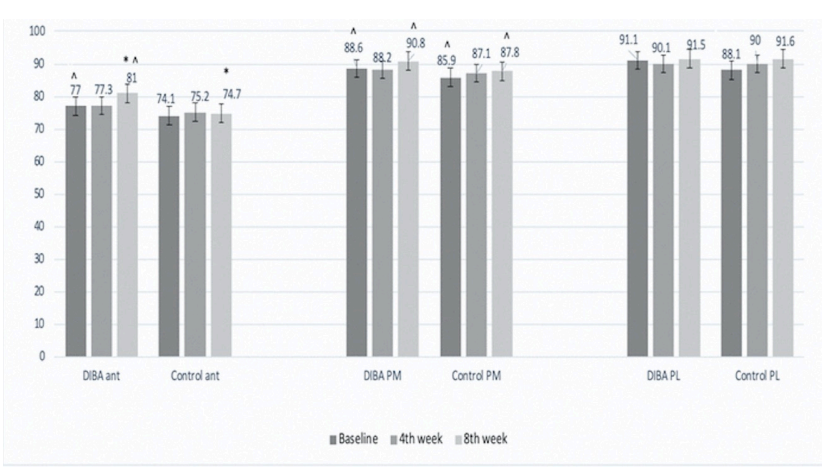

Figure 3. Comparison of Y-Balance test between and within groups.

\footnotetext{
*: Significant difference between groups $p<0.05$

${ }^{\wedge}$ : Significant difference within groups $\mathrm{p}<0.05$
}

was engaging for participants during the eight-week training program. This eight-week duration has also been suggested by Brachmann et al. ${ }^{25}$ for detectable change in balance ability, indeed the results of the current study did not find any significant difference between groups for test scores at the end of the $4^{\text {th }}$ week.

Performing an exercise program on antero-posterior foot platform that could be perturbed during combined activities was more effective in affecting the anterior reach component of YBT than other balance tools. The anterior reach 
of the DIBA group showed a 5.19\% improvement while anterior reach of the control group showed only a \% $0.8 \mathrm{im}-$ provement. On the other hand, medial and lateral component scores of the YBT did not demonstrate significant differences between groups (Posteromedial DIBA group $2.48 \%$, control group $0.8 \%$, posterolateral DIBA group $0.43 \%$, control group $3.9 \%)$. This may be because the mediolateral perturbation done by the DIBA is not as large as that which occurs in the antero-posterior perturbation. This could suggest that mediolateral perturbation of trunk stability may be increased when the two feet are apart from each other. Consequently, healthy participants without balance deficits may not show improvement in their balance. It would be worthwhile to study this exercise progression for balance training program using the DIBA on people with lower extremity injuries.

Assessment of static balance using FBT showed that number of falls for the DIBA group was less when compared to control group at the end of $8^{\text {th }}$ week. This result may indicate improvement in proprioceptive input that allows for accurate motor responses that function to keep the body's center of mass over the base of support, which may have been due to the dynamic behavior of the DIBA. Improving proprioceptive acuity of the muscular structures of lower extremity muscles using a dynamic balance training system may improve the afferent contributions of proprioceptors in the muscles such as muscle spindles and Golgi tendon organ, positively affecting balance ability. ${ }^{26}$ This assertion was not directly studied in this research, however.

Despite the feet being fixed on movable foot platforms when using the DIBA, the distal end of the lower extremity still moves antero-posterior and mediolateral directions, which may be considered an open kinetic chain. However, these movements are not completely unconstrained, as maintaining of balance over the DIBA requires movement of proximal segments. Therefore, the combination of open and closed kinetic chain exercises with the DIBA may be more advantageous than the other balance equipment.

Poor performance on the YBT is associated with an increased risk of variety of lower extremity injuries. Especially, 'poor performance' in anterior direction of the YBT (total reach direction and asymmetry), has been shown to have the most consistent relationship with increased injury risk. $^{27,28}$ Anterior (ANT) reach distance asymmetries greater than $4 \mathrm{~cm}$ are associated with a 2.3 to 2.5-time greater risk of lower extremity injury. ${ }^{29}$ In the current study, healthy subjects in the control group had a mean anterior reach asymmetry of $6.3 \mathrm{~cm}$, which is greater than this threshold. This may indicate that the sedentary subjects in the current study were at an increased risk of sustaining a lower extremity injury.

The importance of being able to produce large ranges of hip flexion is supported by the kinetic models for posterior reach distances including hip extensor moments for both posterior medial (PM) and posterior lateral (PL) reaches, and by other studies ${ }^{25,30}$ which show that hip extension strength is strongly correlated with posterior reach perfor-

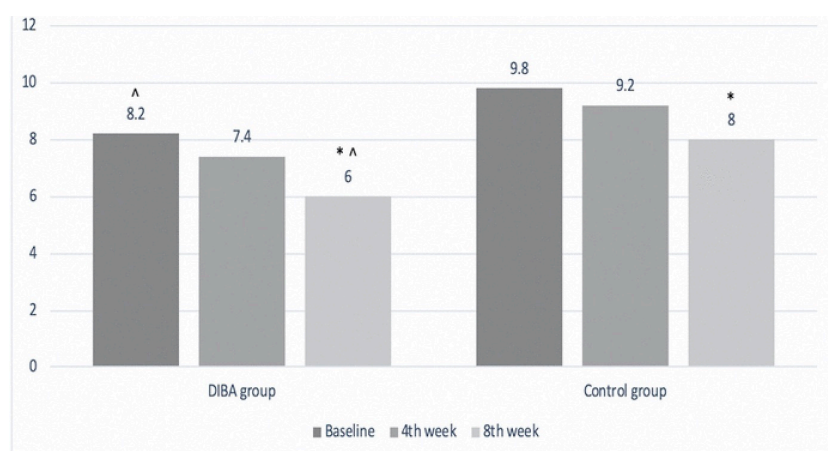

\section{Figure 4. Comparison of Flamingo balance test between and within groups.}

$*$ : Significant difference between groups $\mathrm{p}<0.05$
$\wedge$ : Significant difference within groups $\mathrm{p}<0.05$

mance. The knee extensor and hip abductor moment explained variance in ANT and PM reaches, while the hip extensor moment explained variance in PL and PM reaches. ${ }^{29,30}$

This study was limited to the measurement of young healthy individuals; therefore, the results may differ for subjects of different ages and in the presence of clinical conditions. Second, testing of muscle strength that would be worthwhile to investigate to discern whether a change in muscle strength affected measures static and dynamic balance. Thirdly, the study design did not allow any conclusions to be drawn about level of the muscular system at which any adaptations occurred or if these would be transferred to sports movements. Finally, the majority of participants were first year physiotherapy students and therefore may have had some previous exposure to balance training, the effect of which is not known. Tasks were repeated and therefore it is possible that fatigue affected the overall performance. It is possible that a learning effect may have been present. We think that the DIBA would be reliable for doing balance exercises within a healthy population and provide a reference for further clinical studies.

\section{CONCLUSION}

The results of the current study indicate that the DIBA may improve balance better than balance exercises using standard balance training tools such as the BOSU, balance boards, wobble board, and a soft cushion block. Clinical improvements in dynamic measures of postural control provide an insight into the use of an alternative form of functional balance training using the DIBA with healthy subjects.

Submitted: December 04, 2020 CDT, Accepted: April 17, 2021

CDT 


\section{REFERENCES}

1. McKeon PO, Ingersoll CD, Kerrigan DC, Saliba E, Bennett BC, Hertel J. Balance training improves function and postural control in those with chronic ankle instability. Med Sci Sports Exerc.

2008;40(10):1810-1819. doi:10.1249/mss.0b013e3181 $\underline{7 \mathrm{e} 0 \mathrm{f} 92}$

2. Gonçalves DFF, Ricci NA, Coimbra AMV. Equilíbrio funcional de idosos da comunidade: comparação em relação ao histórico de quedas. Braz J Phys Ther. 2009;13(4):316-323. doi:10.1590/s1413-35552009005 $\underline{000044}$

3. Michell TB, Ross SE, Blackburn JT, et al. Functional balance training, with or without exercise sandals, for subjects with stable or unstable ankles. J Athl Train. 2006;41(4):393-398.

4. Paillard T, Noe F, Riviere T, et al. Postural performance and strategy in the unipedal stance of soccer players at different levels of competition. J Athl Train. 2006;41(2):172-176.

5. Rugelj D. The effect of functional balance training in frail nursing home residents. Arch Gerontol Geriatr. 2010;50(2):192-197. doi:10.1016/j.archger.2009.03.00 9

6. Williams GN, Allen EJ. Rehabilitation of syndesmotic (high) ankle sprains. Sports Health. 2010;2(6):460-470. doi:10.1177/1941738110384573

7. Williams J, Bentman S. An investigation into the reliability and variability of wobble board performance in a healthy population using the SMARTwobble instrumented wobble board. Phys Ther Sport. 2014;15(3):143-147. doi:10.1016/j.ptsp.2013.0 $\underline{8.003}$

8. Haksever B. Comparison of wii therapy and conventional rehabilitation protocol after ACL reconstruction with hamstring tendon greft. 2012.

9. Son SM, Park MK, Lee NK. Influence of resistance exercise training to strengthen muscles across multiple joints of the lower limbs on dynamic balance functions of stroke patients. J Phys Ther Sci. 2014;26(8):1267-1269. doi:10.1589/jpts.26.1267

10. Oddsson LIE, Karlsson R, Konrad J, Ince S, Williams SR, Zemkova E. A rehabilitation tool for functional balance using altered gravity and virtual reality. J Neuroeng Rehabil. 2007;4(1):25-25. doi:10.11 86/1743-0003-4-25
11. Galeano D, Brunetti F, Torricelli D, Piazza S, Pons JL. A tool for balance control training using muscle synergies and multimodal interfaces. BioMed Research International. 2014:1-13. doi:10.1155/2014/565370

12. Bhat R, Moiz JA. Comparison of dynamic balance in collegiate field hockey and football players using star excursion balance test. Asian J Sports Med. 2013;4(3):221-229. doi:10.5812/asjsm.34287

13. Haskell WL, Lee IM, Pate RR, et al. Physical activity and public health. Med Sci Sports Exerc. 2007;39(8):1423-1434. doi:10.1249/mss.0b013e31806 $\underline{16 \mathrm{~b} 27}$

14. Leavitt MO. Physical Activity Guidelines for Americans. USA: Department of Health and Human Services; 2008.

15. Plisky PJ, Rauh MJ, Kaminski TW, Underwood FB. Star excursion balance test as a predictor of lower extremity injury in high school basketball players. $J$ Orthop Sports Phys Ther. 2006;36(12):911-919. doi:1 0.2519/jospt.2006.2244

16. Stabilometry of the flamingo balance test. Presented at the: 14 International Symposium on Biomechanics in Sports; 1996; Madeira, Portugal.

17. Howe TE, Rochester L, Jackson A, Banks PM, Blair VA. Exercise for improving balance in older people. Howe TE, ed. Cochrane Database of Syst Rev. October 2007. doi:10.1002/14651858.cd004963.pub2

18. Pollock AS, Durward BR, Rowe PJ, Paul JP. What is balance? Clin Rehabil. 2000;14(4):402-406. doi:10.119 1/0269215500cr342oa

19. Riemann BL, Lephart SM. The sensorimotor system, part I: The physiologic basis of functional joint stability. J Athl Train. 2002;37(1):71-79.

20. Karanjia PN, Ferguson JH. Passive joint position sense after total hip replacement surgery. Ann Neurol. 1983;13(6):654-657. doi:10.1002/ana.410130612

21. Proske U, Gandevia SC. The proprioceptive senses: their roles in signaling body shape, body position and movement, and muscle force. Physiol Rev. 2012;92(4):1651-1697. doi:10.1152/physrev.00048.201 1

22. Aman JE, Elangovan N, Yeh IL, et al. The effectiveness of proprioceptive training for improving motor function: a systematic review. Front Hum Neurosci. 2014;8:1075. 
23. Howe TE, Rochester L, Neil F, Skelton DA, Ballinger C. Exercise for improving balance in older people. Cochrane Database Syst Rev. 2011;2011(11):CD004963. doi:10.1002/14651858.cd00 4963.pub3

24. Petró B, Papachatzopoulou A, Kiss RM. Devices and tasks involved in the objective assessment of standing dynamic balancing - A systematic literature review. Gard SA, ed. PLoS ONE. 2017;12(9):e0185188. doi:10.1371/journal.pone.0185188

25. Brachman A, Kamieniarz A, Michalska J, Pawłowski M, Słomka KJ, Juras G. Balance training programs in athletes - A systematic review. J Hum Kinet. 2017;58(1):45-64. doi:10.1515/hukin-2017-008 $\underline{8}$

26. Wang H, Ji Z, Jiang G, Liu W, Jiao X. Correlation among proprioception, muscle strength, and balance. J Phys Ther Sci. 2016;28(12):3468-3472. doi:10.1589/i pts.28.3468
27. Miller MM, Trapp JL, Post EG, et al. The effects of specialization and sex on anterior Y-balance performance in high school athletes. Sports Health. 2017;9(4):375-382. doi:10.1177/1941738117703400

28. Gonell AC, Romero JAP, Soler LM. Relationship between the Y-balance test scores and soft tissue injury incidence in a soccer team. Int J Sports Phys Ther. 2015;10:955-966.

29. Smith CA, Chimera NJ, Warren M. Association of Y-balance test reach asymmetry and injury in Division I athletes. Med Sci Sports Exerc. 2015;47(1):136-141. doi:10.1249/mss.0000000000000 $\underline{380}$

30. Nelson S, Wilson CS, Becker J. Kinematic and kinetic predictors of Y-balance test performance. Int J Sports Phys Ther. 2021;16(2):371-380. 